\title{
Investigation of Scattered Radiation Dose at the Door of a Radiotherapy Vault When the Maze Intersects the Primary Beam
}

\author{
Sean Michael Tanny, Nicholas Niven Sperling, E. Ishmael Parsai \\ Department of Radiation Oncology, Division of Medical Physics, University of Toledo Medical Center, Toledo, \\ Ohio, USA \\ Email: e.parsai@utoledo.edu
}

Received 27 January 2015; accepted 13 February 2015; published 25 February 2015

Copyright $@ 2015$ by authors and Scientific Research Publishing Inc.

This work is licensed under the Creative Commons Attribution International License (CC BY).

http://creativecommons.org/licenses/by/4.0/

(c) () Open Access

\begin{abstract}
NCRP 151 provides very detailed examples demonstrating the necessary concerns for shielding a conventional radiotherapy vault with a maze where the useful beam is parallel to the maze. However, it provides little guidance on how to properly shield a vault with the maze-wall acting as part of a compound primary barrier. We have modeled a new radiotherapy vault with this configuration and assessed the additional photon shielding burden at the door with MCNP5. MCNP simulations demonstrated an increase in overall photon shielding burden at the door relative to calculations that only consider photon workloads presented in NCRP 151. Two additional components of scattered radiation are considered and methods for calculation are presented.
\end{abstract}

\section{Keywords}

\section{Radiation Protection, Radiation Shielding, Scatter, Monte Carlo}

\section{Introduction}

NCRP Report 151 lays out the broad considerations for shielding a megavoltage linear accelerator to achieve reasonably low levels of radiation [1]. An example is provided for the medical physicist to accurately calculate the shielding requirements for a radiation therapy vault with a maze. However, the example for a conventional linear accelerator vault presented in NCRP 151 only applies to a layout where the useful beam does not intersect the maze barrier. Facilities utilizing a design where the primary beam does intersect the maze barrier require additional guidance.

Alternative vault designs are often the product of trying to minimize shielding material within tight space restrictions. The options available to the shielding designer are a direct shielded door, door with a shortened maze,

How to cite this paper: Tanny, S.M., Sperling, N.N. and Parsai, E.I. (2015) Investigation of Scattered Radiation Dose at the Door of a Radiotherapy Vault When the Maze Intersects the Primary Beam. Journal of Modern Physics, 6, 141-149. 
or a configuration with the useful beam intercepting a standard maze. A direct shielded door or shortened maze design for a high-energy accelerator (>10 MV) requires considerable shielding material to provide adequate attenuation from direct photo-neutrons and gamma capture photons. Designing a longer maze barrier helps to attenuate these effects, but requires more shielding material. Allowing adjacent vaults to share barriers minimizes the amount of shielding material for a facility, which can lead to having the primary beam incident on the maze depending on the facility geometry. With a beam incident on the maze, it is desirable to split the primary barrier shielding between the maze barrier and the maze wall, making the maze barrier as thin as possible to minimize dose at the door and the necessary volume of concrete required for the facility. Splitting the primary barrier into a compound barrier introduces new considerations, namely primary beam that is scattered from the maze barrier to the door, primary transmission through the maze barrier incident on the door, and primary transmission through the maze barrier that is then scattered to the door by the far maze wall.

A variant of this problem was previously investigated by Facure et al. where they studied the effects of photon dose-equivalent at the vault door from "unusual" gantry orientations [2]. They found that an increase by up to a factor of 7 for photon dose-equivalent could be achieved by orienting the gantry at $45^{\circ}$ to the maze normal, but that photon dose-equivalent was reduced by approximately a factor of 2 by pointing the gantry directly towards the maze. However, their study did not attempt to define a method to calculate this photon dose. Additionally, they did not simulate higher energy accelerators capable of producing beams $>10 \mathrm{MV}$.

Biggs explored the necessary thickness of door shielding for 4 and $10 \mathrm{MV}$ accelerators when the primary beam intercepted a standard maze and a shortened maze [3]. They concluded that the relative accuracy of the NCRP 49 and NCRP 51 formalisms provided a conservatively safe estimate of the photon dose-equivalent, but that the dose-equivalent at the door was higher for situations where the beam was perpendicular to the maze. This increase in dose-equivalent was larger for the higher-energy beam. There was also a greater variation in dose-equivalent at different positions across the door than with a conventional vault.

Zavgorodni evaluated scattered dose transmitted through under-shielded ceilings incident on adjacent buildings in 2001, similar to the situation of the maze barrier [4]. This investigation included both simulation and direct measurement, demonstrating agreement within $40 \%$. They determined that a power-law fit for scatter fractions was adequate to model the measured dose for all energies. Their work did include a method for calculating the scattered dose from a broad beam, but the scatter fractions were calculated over large distances (5 - 10 meters) whereas the door is commonly situated within half that distance. With the area of interest much closer to the scattering source, we no longer can approximate the source as a point. Effects from an elongated scattering source will be more pronounced at the shorter distances involved when shielding the door.

Our goal is to identify how the beam intercepting the maze barrier contributes to the photon shielding workload at the door. We have developed a model consistent with the derivation presented by Zavgorodni and have created a Monte Carlo simulation to determine the appropriate shielding in this situation [4]. The additional photon workload has been compared to the photon dose calculated using the existing formalisms presented in NCRP 151 for leakage scatter, patient scatter, leakage transmission, and wall scatter [1].

\section{Methods}

\subsection{Vault Layout}

Figure 1 presents the layout of the vault, with the relevant dimensions listed in Table 1 . The primary beam is normally incident on the maze barrier, but is not directly incident on the vault door. The bold arrows on Figure 1 depict the paths of scattered radiation to the door. The path for leakage transmission from isocenter through the maze barrier to the door centerline has been omitted for clarity, but should still remain a consideration for shielding in this configuration.

\subsection{Monte Carlo Model}

A Monte Carlo model of the vault was designed using the MCNP5 code from Los Alamos National Laboratory [5]. Only photon transport was modeled to determine the additional scatter dose. Neutron production and additional gamma capture workload were not included in the simulation as they can be calculated using the formalisms in NCRP 151, detailed in the following section [1]. The photon spectrum used was taken from a machine model in the Pinnacle TPS (Philips Radiation Oncology Systems, Madison 5520 Nobel Dr Ste 125. Fitchburg, 


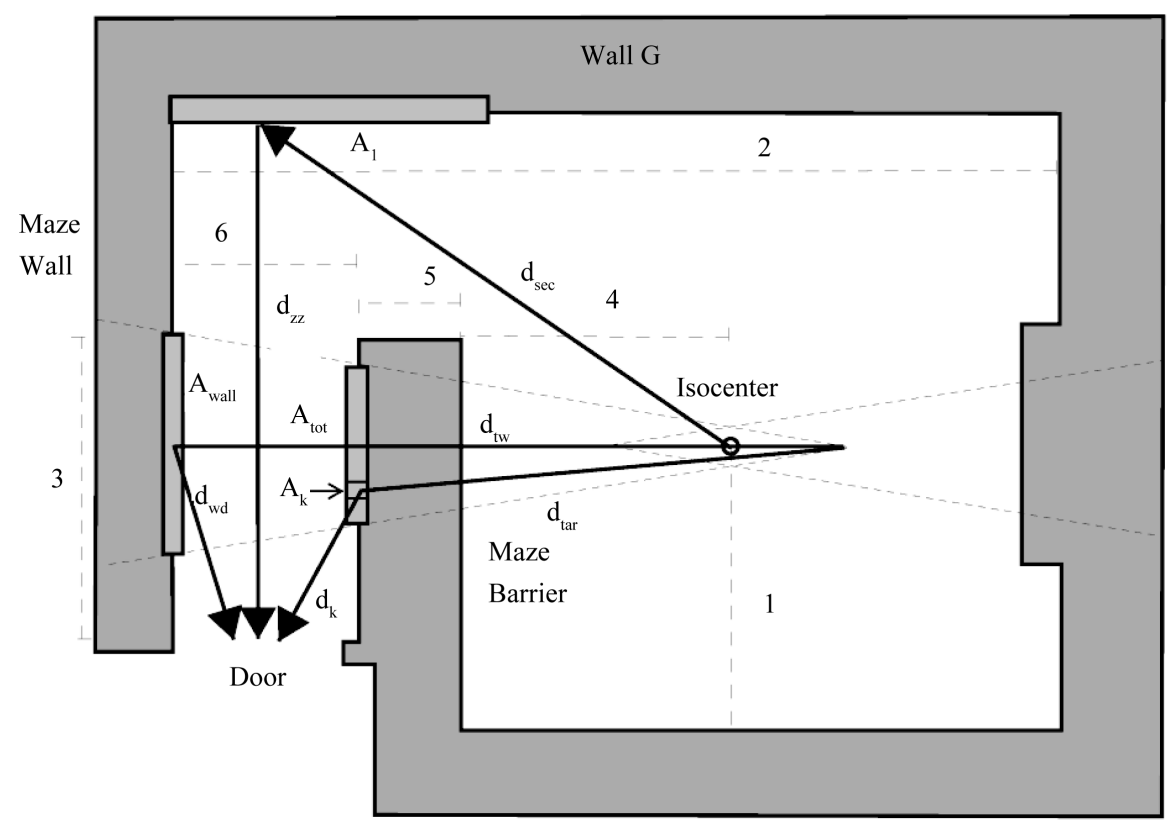

Figure 1. Diagram of simulated vault. Numbered segments correspond to lengths in Table 1. Thick arrows show potential paths for radiation to reach the door. The path for leakage transmission from isocenter to door centerline has been omitted for clarity.

Table 1. Vault dimensions for MCNP simulation and shielding design calculations.

\begin{tabular}{lcc}
\hline Dimension & Number on Figure 1 & Distance (m) \\
\hline Isocenter to Wall G & 1 & 4.42 \\
Outer Boundary of Door to Far Edge of Maze & 2 & 3.97 \\
Outer Boundary of Door to Mid-line of Field & 3 & 2.60 \\
Isocenter to Maze Barrier & 4 & 3.28 \\
Maze Barrier Thickness & 5 & 1.22 \\
Maze Width & 6 & 1.98 \\
\hline
\end{tabular}

Wisconsin 53711-4948). Spectra for 6 and $18 \mathrm{MV}$ were run. These were compared with the accepted spectra from Mohan et al. and determined to be comparable [6]. A planar source was created at the opening of the machine head directly below the multi-leaf collimator (MLC) with an opening angle corresponding to a $40 \times 40$ $\mathrm{cm}^{2}$ field at isocenter. A $40 \times 40 \times 40 \mathrm{~cm}^{3}$ water phantom was centered about isocenter (i.e. the center of the phantom was placed at isocenter). Simulations were run for $10^{10}$ histories. Photon energy cutoffs were set to 30 $\mathrm{keV}$ to decrease simulation time. Preliminary runs showed less than a $2 \%$ difference for simulations with a 10 $\mathrm{keV}$ photon cutoff.

The maze barrier was divided lengthwise into $11 \mathrm{~cm}$ thick cells, with the $11 \mathrm{~cm}$ furthest from isocenter being divided into $10 \mathrm{~cm}$ long by $20 \mathrm{~cm}$ high segments. The relative photon-importance within each layer of the maze barrier was increased from 1 - 10. The photon importance for cells beyond the maze barrier was maintained at 10. The tally region beyond the door was subdivided into $20 \times 10 \times 2 \mathrm{~cm}^{3}$ cells that were vertically centered along the beam line. Tallies were placed beyond each barrier and beyond the door, as well as a $20 \times 20 \times 1 \mathrm{~cm}^{3}$ scoring plane centered about isocenter at $20 \mathrm{~cm}$ depth within the water phantom. Dose at isocenter was then scaled appropriately using the TMR (20) to determine dose at $d_{\max }$.

Energy deposition was tracked with the F6 tally, a track-length estimator of energy deposition averaged over the mass of the cell. Tally contributions at the door were flagged with relative contributions from cells inside the maze barrier to determine the amount of maze-scattered radiation incident on the door. Energy deposition within the second half of the maze barrier was tallied and used to fit the HVL of concrete as a verification of source at- 
tenuation. The second half of the maze barrier was used to avoid any variation between initial and equilibrium HVLs. The simulated HVL was compared to a calculated HVL using TVLs for concrete presented by NCRP $151[1]$.

Scatter-fractions were derived from the relative contributions of cells in the outer portion of the maze barrier to the relative contribution of dose in the door. These were normalized to the strength at $1 \mathrm{~m}$ from the maze barrier via by correcting for inverse square effects. Scatter intensity was renormalized by dividing the ratio of the simulated field size to a $400 \mathrm{~cm}^{2}$ field. This process is summarized in Equation (1),

$$
a_{j}\left(\theta_{k}\right)=\frac{D_{\text {Cell }_{k}}}{D_{\text {Maze }_{k}}}\left(\frac{d_{k}}{1 \mathrm{~m}}\right)^{2}\left(\frac{400 \mathrm{~cm}^{2}}{F}\right)
$$

Simulated concrete material was a density of $2.35 \mathrm{~g} \cdot \mathrm{cm}^{-3}$ and a composition by weight of $2.2 \%$ hydrogen, $57.5 \%$ oxygen, $1.5 \%$ sodium, $0.12 \%$ magnesium, $2.0 \%$ aluminum, $30.5 \%$ silicon, $1.0 \%$ potassium, $4.3 \%$ calcium, and $0.6 \%$ iron [7]. This composition is somewhat different from the simulated materials used by Biggs, and Facure et al. Biggs cites their concrete composition as being from NCRP Report 31 and Facure et al. utilized compositions used during prior work [2] [3]. The compositions differ in the relative composition by weight, along with minor additives, such as sodium, magnesium, and potassium, but all works utilize a density of 2.35 $\mathrm{g} \cdot \mathrm{cm}^{-3}$.

\subsection{NCRP Formalism for Door Workload}

The method for calculating the patient-scatter workload, $H_{P S}$, at the vault door is presented in Equation (2),

$$
H_{P S}=\sum_{j} \frac{W_{j} U_{\text {Maze }} a_{j}(\theta) \alpha_{1} A_{1}}{\left(d_{s c a} d_{s e c} d_{z z}\right)^{2}} \frac{F}{400}
$$

where $a_{j}(\theta)$ is the scatter fraction of patient-scattered radiation at angle $\theta$ and energy $j, W_{j}$ is the primary workload for energy $j, U_{\text {Maze }}$ is the use factor for the maze, $F$ is the maximum field size in $\mathrm{cm}^{2}$ at isocenter, $A_{1}$ is the area of wall $G$ visible from the maze entrance, $\alpha_{1}$ is the concrete albedo for wall $G, d_{s c a}$ is the distance from target to patient (typically $1 \mathrm{~m}$ ), $d_{s e c}$ is the distance from patient to wall $G$, and $d_{z z}$ is the distance from wall $G$ to the maze entrance [1].

Leakage transmission and leakage scatter, $H_{L T}$ and $H_{L S}$, are calculated using Equations (4) and (5) respectively.

$$
\begin{aligned}
& M_{j}=10^{-\left(\frac{t-\mathrm{TVL}_{1}}{\mathrm{TVL}_{2}}+1\right)} \\
& H_{L T}=\sum_{j} \frac{W_{j} L U_{\text {Maze }} M_{j}}{\left(d_{L}\right)^{2}} \\
& H_{L S}=\sum_{j} \frac{W_{j} L U_{\text {Maze }} \alpha_{1} A_{1}}{\left(d_{\text {sec }} d_{z z}\right)^{2}}
\end{aligned}
$$

$L$ is the maximum allowable amount of head leakage, taken as $0.001, M_{j}$ is the full maze barrier attenuation factor given by Equation (3), $d_{L}$ is the distance from the isocenter to the center of the door, $t$ is the thickness of the maze barrier including slant thickness, and the other variables are equivalent to what was defined above for patient scattered radiation [1].

The primary wall-scattered radiation component discussed in NCRP 151 and McGinley 2002 is not present in the current configuration, and is not calculated here [1] [8].

\subsection{Additional Photon Components for Compound Barrier Configuration}

We began with the assumption that the maze barrier by itself is not sufficiently thick to reduce the dose rate in the maze to below the desired shielding goal outside of the vault. A certain fraction of the attenuated radiation from the primary beam will be scattered outside of the primary beam by the maze barrier. At a point in the maze barrier, this scattered radiation can escape the remaining shielding material. Therefore, the material will act more as a scattering source than an attenuator for points outside of the useful beam. This should happen at one 
mean free path (MFP) from the left edge of the maze barrier in Figure 1. We can determine the MFP from the half-value layer (HVL) by Equation (6),

$$
\mathrm{MFP}=\frac{\mathrm{HVL}}{\ln (2)}
$$

We assume that radiation which makes it to this point in the maze barrier escapes without further attenuation.

Radiation reaching within one MFP of the left edge of the maze barrier has a high likelihood of scattering and then escaping the barrier. Typically there is one predominant angle for calculating the scatter workload, but our situation applies to an extended source at close range, thus increasing the range of relevant scattering angles considerably. A more accurate method to calculate the scattered dose is to divide the irradiated area into subsegments and calculate the contribution from each one. We utilize the scatter fractions respective to the angle from each subsection of the maze barrier to the door, scaled by the area of the maze barrier subsection over the total irradiated area of the maze. The effective source strength is given by the workload of each energy at isocenter multiplied by the maze attenuation factor, given by Equation (7). This is summed over the total area of the maze exposed to primary radiation. The maze-scatter workload for the door, $H_{M S}$, can be calculated as shown in Equation (8),

$$
\begin{gathered}
B_{j}=10^{-\left(\frac{t-\mathrm{TVL}_{1}-\mathrm{MFP}_{2}}{\mathrm{TVL}_{2}}+1\right)} \\
H_{M S}=\sum_{j k} \frac{W_{j} B_{j} a_{j}\left(\theta_{k}\right) U_{\mathrm{Maze}} A_{k}}{\left(d_{\text {tar }} d_{k}\right)^{2} A_{\text {tot }}} \frac{F}{400}
\end{gathered}
$$

where $a_{j}\left(\theta_{k}\right)$ is the scatter fraction for the $k$-th maze section of the $j$-th energy, $t$ is the thickness of the maze barrier, $A_{k}$ is the area of the $k$-th maze section, $A_{\text {tot }}$ is the total area of the far maze barrier irradiated, $d_{t a r}$ is the distance from the target to the far side of the maze, and $d_{k}$ is the distance from the $k$-th maze section to the point in the door.

Primary transmission scattered from the maze wall is calculated using Equation (9),

$$
H_{W S}=\sum_{j} \frac{W_{j} M_{j} U_{\text {Maze }} \alpha_{\text {Wall }} A_{\text {wall }}}{\left(d_{W D} d_{T W}\right)^{2}}
$$

where $W_{j}$ and $U_{\text {Maze }}$ are as defined above, $M_{j}$ is given by Equation (3), $A_{\text {Wall }}$ is the irradiated area of the maze wall, $\alpha_{W a l l}$ is the concrete albedo of the maze wall, $d_{T W}$ is the distance from the target to the maze wall, and $d_{W D}$ is the distance from the maze wall to the door.

\subsection{Total Photon Workload at the Door}

The scattered photon shielding workload at the door for the gantry directed at the maze, $H_{\text {Maze }}$, can be calculated as the summation of these different components, as shown in Equation (10),

$$
H_{\text {Maze }}=H_{P S}+H_{L S}+H_{L T}+H_{M S}+H_{W S}
$$

Unlike standard vault design where there is a greater symmetry between radiation dose at the door for a single gantry angle and overall radiation dose at the door, this current vault design is very sensitive to the direction of the radiation beam. Rather than multiplying $H_{\text {Maze }}$ by a single factor to get $H_{\text {Door }}$, only the patient scatter, leakage scatter, and leakage transmission components can be extended to other gantry angles with the standard multiplier of 2.64 [1] [8]. We do not explore the accuracy of this empirical factor in this study. The contribution of maze-scatter and wall-scatter should be negligible when the gantry is not directed towards the maze. Thus, the total photon workload at the door, $H_{\text {Door }}$, can be calculated by Equation (11),

$$
H_{\text {Door }}=2.64 \times\left(H_{P S}+H_{L S}+H_{L T}\right)+H_{M S}+H_{W S}
$$

\section{Results}

\subsection{Monte Carlo}

Figure 2 demonstrates the attenuation through the maze barrier. HVLs from the simulation were $9.9 \mathrm{~cm}$ and 


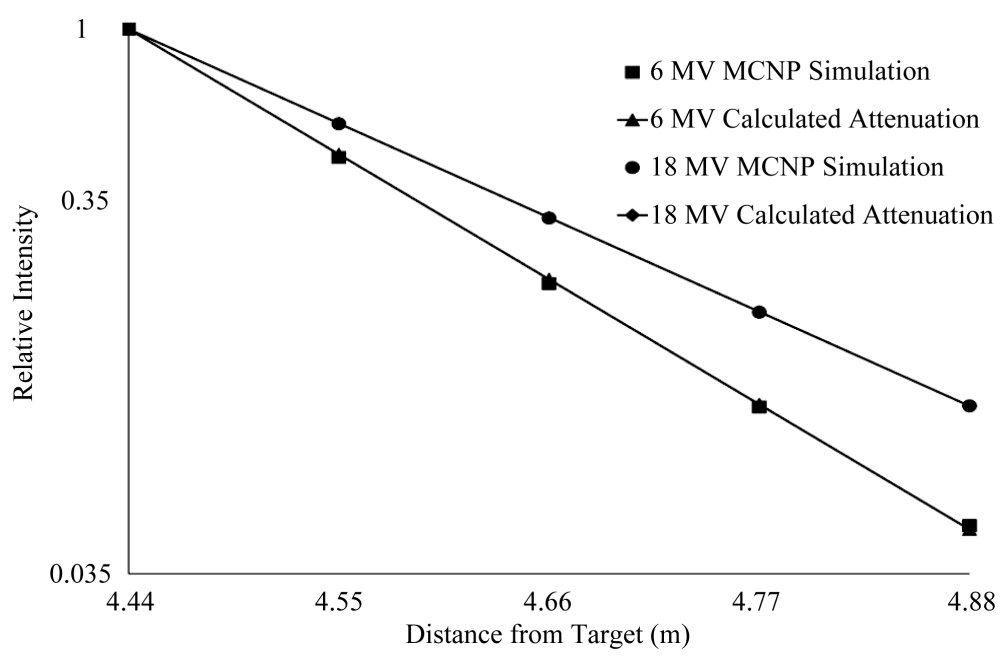

Figure 2. Simulated vs. calculated HVLs within the maze barrier. Normalized to intensity halfway through the maze barrier.

$13.1 \mathrm{~cm}$ compared to $10.0 \mathrm{~cm}$ and $13.1 \mathrm{~cm}$ for concrete HVLs calculated from NCRP 151 for 6 and $18 \mathrm{MV}$, respectively [1]. These are demonstrated in Figure 2. The mean difference between simulated attenuation and the fit value was $0.97 \%$ and $0.23 \%$ for 6 and $18 \mathrm{MV}$, while the statistical uncertainty of each tally within the maze barrier was less than $0.05 \%$. Of the additional photon dose in the door, $90.8 \% \pm 2.8 \%$ was contributed by the final $11 \mathrm{~cm}$ of the maze barrier for both 6 and $18 \mathrm{MV}$.

The maximum dose in the door from maze-scattered radiation relative to the dose at isocenter was $6.81 \times 10^{-9}$ $\pm 17.6 \%$ for $6 \mathrm{MV}$. The maximum dose in the door per Gray at isocenter was $5.20 \times 10^{-7} \pm 0.3 \%$ and $5.84 \times 10^{-7}$ $\pm 0.2 \%$ for 6 and $18 \mathrm{MV}$, respectively. The location of maximum dose in the door was at the corner furthest from the maze barrier. The tallies nearest the maze barrier recorded $80 \% \pm 0.4 \%$ of the maximum dose within the door for 6 MV. Scatter fractions from concrete are presented in Figure 3 and Figure 4 and numerically in Table 2. These are calculated from two of the tallies within the door. The range of angles covered extends from 16 to 78 degrees, with most of these being covered by both tallies.

\subsection{NCRP Calculation Formalism}

Table 3 presents results from an NCRP style calculation for the relative contributions of photon dose at the door in Gray per Gray delivered to isocenter. Patient scattering and wall scatter albedos were taken as $0.5 \mathrm{MeV}$ effective energy. Use of this energy for patient scatter is recommended by NCRP 151 [1]. Wall scatter within the maze is dealing with a significantly lower effective energy once the primary beam has been transmitted through the barrier. Gamma-capture photons were not simulated, but were calculated for $18 \mathrm{MV}$. These dominate the total photon shielding burden for high-energy, reaffirming that scattered radiation doses can be safely neglected when shielding gamma-capture photons. Table 4 presents equivalent dose rates at the door for a machine with a weekly workload of 1000 Gray.

\section{Discussion}

The scattering fractions derived from the MCNP simulation require some attention. There is similar data for scattering phenomenon from the patient, but patient scatter fractions are not expected to be ideal for this situation. There are several differences between the situation of patient scatter and the situation presented here. The $Z_{\text {eff }}$ of concrete can vary between $12-13$, while the $Z_{\text {eff }}$ of tissue is $\sim 8$. The spectral changes introduced by the greater attenuation of the maze barrier ( 1 - 3 TVLs) relative to the patient (1 - 2 HVLs) will also cause some differences in the scattering properties of the beam. Beyond that, the scattering surface is flat when simulating concrete scatter factors, whereas patient scatter factors were derived from simulation of a spherical phantom. The side-scatter fractions derived by Zavgorodni share more of the physics in common with maze scatter [4]. However, the side-scatter fractions are an order of magnitude greater than the patient scatter fractions [4] [9]. 


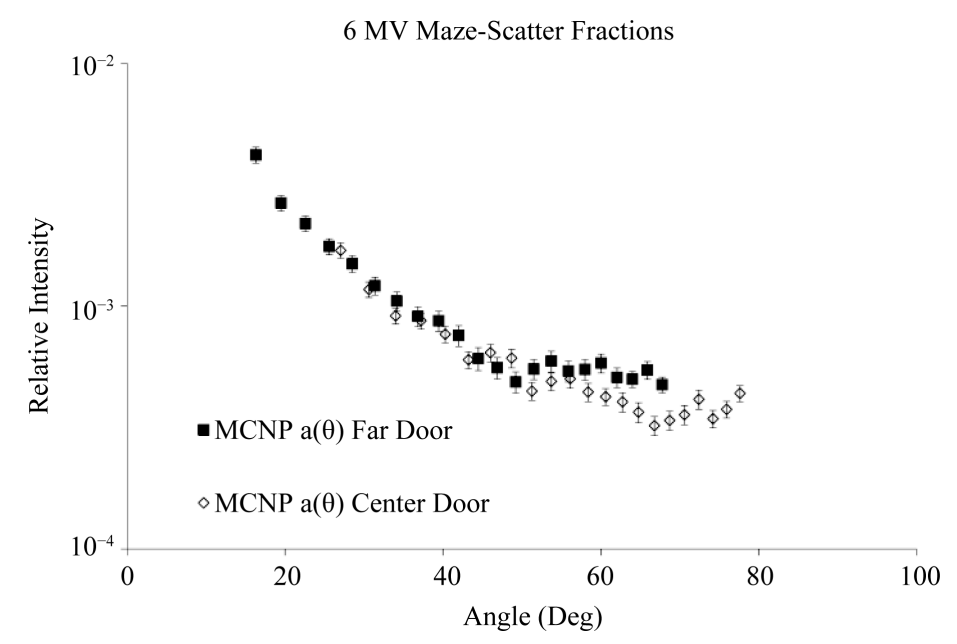

Figure 3. Simulated 6 MV maze scatter fractions.

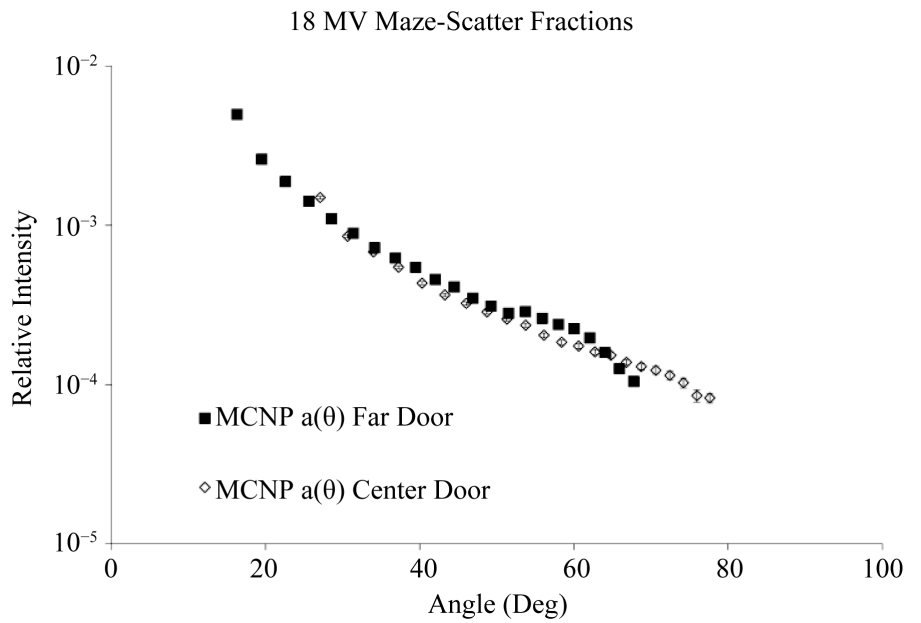

Figure 4. Simulated $18 \mathrm{MV}$ maze scatter fractions.

Table 2. Simulated maze scatter fractions for 6 and $18 \mathrm{MV}$.

\begin{tabular}{ccc}
\hline Angle (degrees) & 6 MV concrete & 18 MV concrete \\
\hline 20 & $1.42 \times 10^{-03} \pm 1.06 \times 10^{-04}$ & $1.44 \times 10^{-03} \pm 2.43 \times 10^{-05}$ \\
30 & $6.74 \times 10^{-04} \pm 5.51 \times 10^{-05}$ & $4.95 \times 10^{-04} \pm 1.09 \times 10^{-05}$ \\
45 & $2.99 \times 10^{-04} \pm 3.21 \times 10^{-05}$ & $1.98 \times 10^{-04} \pm 6.21 \times 10^{-06}$ \\
60 & $2.74 \times 10^{-04} \pm 2.56 \times 10^{-05}$ & $1.10 \times 10^{-04} \pm 4.81 \times 10^{-06}$ \\
75 & $1.80 \times 10^{-05} \pm 1.46 \times 10^{-05}$ & $4.77 \times 10^{-05} \pm 3.65 \times 10^{-06}$ \\
\hline
\end{tabular}

Both of these data sets demonstrate larger scattering fractions than our simulations, but we still find that the contribution is not negligible.

Radiation scattered from the maze wall for $6 \mathrm{MV}$ contributes the greatest amount of dose equivalent per the deterministic calculation. The use of the lower energy dose albedo for $0.5 \mathrm{MeV}$ is appropriate following transmission through a thick barrier. The appropriateness of this factor for thin maze barriers or barriers made of high-density concrete remains a focus for further study. Wall scattered radiation accounts for $43 \%$ of the calculated photon dose equivalent at $6 \mathrm{MV}$, and $84 \%$ of the scattered photon dose equivalent for $18 \mathrm{MV}$. This is relatively low energy scattered radiation. In facilities with machines capable of producing photoneutrons, the large 
Table 3. Relative intensities of sources of photon dose equivalent at the vault door as per an NCRP style calculation. Values are in Gy at the door per Gy at isocenter. Contributions are calculated for the radiation field pointing towards the maze.

\begin{tabular}{ccc}
\hline Photon component & $\mathbf{6 ~ M V}$ & $\mathbf{1 8 ~ M V}$ \\
\hline Patient scatter & $1.68 \times 10^{-8}$ & $1.17 \times 10^{-8}$ \\
Leakage scatter & $1.21 \times 10^{-8}$ & $1.21 \times 10^{-8}$ \\
Leakage transmission & $2.23 \times 10^{-10}$ & $8.31 \times 10^{-10}$ \\
Maze scatter & $3.99 \times 10^{-10}$ & $1.95 \times 10^{-10}$ \\
Wall scatter & $2.27 \times 10^{-8}$ & $1.25 \times 10^{-7}$ \\
Gamma capture & --- & $6.49 \times 10^{-7}$ \\
Total (Excluding gamma capture) & $5.23 \times 10^{-8}$ & $1.49 \times 10^{-7}$ \\
\hline
\end{tabular}

Table 4. Dose equivalents in Sv $\cdot \mathrm{wk}^{-1}$ at the vault door for a machine with a weekly workload of $1000 \mathrm{~Gy}$.

\begin{tabular}{ccccc}
\hline & $\mathbf{6} \mathbf{~ M V}$ calculated & $\mathbf{6}$ MV simulated & $\mathbf{1 8}$ MV calculated & $\mathbf{1 8}$ MV simulated \\
\hline$H_{\text {Maze }}$ & $5.23 \times 10^{-5}$ & $1.30 \times 10^{-4}$ & $1.57 \times 10^{-4}$ & $1.46 \times 10^{-4}$ \\
$H_{\text {Door }}$ & $1.01 \times 10^{-4}$ & -- & $8.41 \times 10^{-4}$ & -- \\
\hline
\end{tabular}

TVL for gamma-capture photons will be enough to effectively shield this lower energy scatter. Additionally, for $18 \mathrm{MV}$, the gamma capture photon dose equivalent is nearly 5 times greater than the scattered photon dose. Thus, for higher energy machines this additional photon dose equivalent can safely be ignored when shielding for gamma capture photons.

The NCRP formalism calculates that the additional shielding workload at the door accounts for $20 \%$ of the photon dose equivalent at $6 \mathrm{MV}$ for a single gantry angle and is approximately $9 \%$ of the overall photon dose for all gantry angles. MCNP simulation results demonstrate a significantly smaller effect. The components of wall scatter and maze scatter only contribute to $3 \%$ of the total photon dose at the door, while all components besides gamma capture contribute 7\%. This supports that scatter doses to the door can still be disregarded for high- energy accelerators in this configuration. While the relative contribution of photon dose-equivalent for $18 \mathrm{MV}$ is lower, the absolute contribution is still larger than the contribution from $6 \mathrm{MV}$. This supports the previous findings of Biggs [10]. Similar to what Biggs found, we also determine that the dose distribution at the door is no longer uniform in this configuration, but varies across the door by $20 \%$ for $6 \mathrm{MV}$. The location of dose maximum is at the corner of the door furthest from the maze barrier, which should be expected as it will have the shallowest scattering angles from maze scatter.

\section{Conclusion}

We have developed a method to account for additional photon dose-equivalent at the maze door for a high-energy therapy accelerator that has been verified by MCNP simulation. This equation utilizes scatter fractions and TVLs of concrete available to the medical physicist from NCRP 151. The agreement between analytic calculations and simulated results demonstrated that the additional dose-equivalent at the vault door was increased from a traditional vault layout, discounting capture gamma photons. However, further study needs to be done to accurately determine the proper scatter fractions for this type of situation. Until such time, our methodology should only be used as an approximation of the additional shielding burden for the door.

\section{References}

[1] National Council on Radiation Protection and Measurements (2005) Structural Shielding Design and Evaluation for Megavoltage X- and Gamma-Ray Radiotherapy Facilities.

[2] Facure, A., Cardoso, S.C., da Rosa, L.A.R. and da Silva, A.X. (2010) Radiation Protection Dosimetry, 138, $251-256$. http://dx.doi.org/10.1093/rpd/ncp245

[3] Biggs, P. (1991) Health Physics, 62, 465-472. http://dx.doi.org/10.1097/00004032-199110000-00001

[4] Zavgorodni, S.F. (2001) Medical Physics, 28, 1926-1930. http://dx.doi.org/10.1118/1.1398302 
[5] X-5 Monte Carlo Team (2005) LA-UR-03-1987. Los Alamos National Laboratory, USA.

[6] Mohan, R., Chui, C. and Lidosfsky, L. (1985) Medical Physics, 12, 592-597. http://dx.doi.org/10.1118/1.595680

[7] Williams III, R.G., Gesh, C.J. and Pagh, R.T. (2006) Pacific Northwest National Laboratory, 14-18.

[8] McGinley, P. (2002) Shielding Techniques for Radiation Oncology Facilities. 2nd Edition, Medical Physics Publishing, Madison.

[9] Taylor, P.L., Rodgers, J.E. and Shobe, J. (1999) Medical Physics, 26, 1442-1446. http://dx.doi.org/10.1118/1.598670

[10] Shobe, J., Rodgers, J.E. and Taylor, P.L. (1999) Health Physics, 76, 25-35. http://dx.doi.org/10.1097/00004032-199901000-00005 
Scientific Research Publishing (SCIRP) is one of the largest Open Access journal publishers. It is currently publishing more than 200 open access, online, peer-reviewed journals covering a wide range of academic disciplines. SCIRP serves the worldwide academic communities and contributes to the progress and application of science with its publication.

Other selected journals from SCIRP are listed as below. Submit your manuscript to us via either submit@scirp.org or Online Submission Portal.
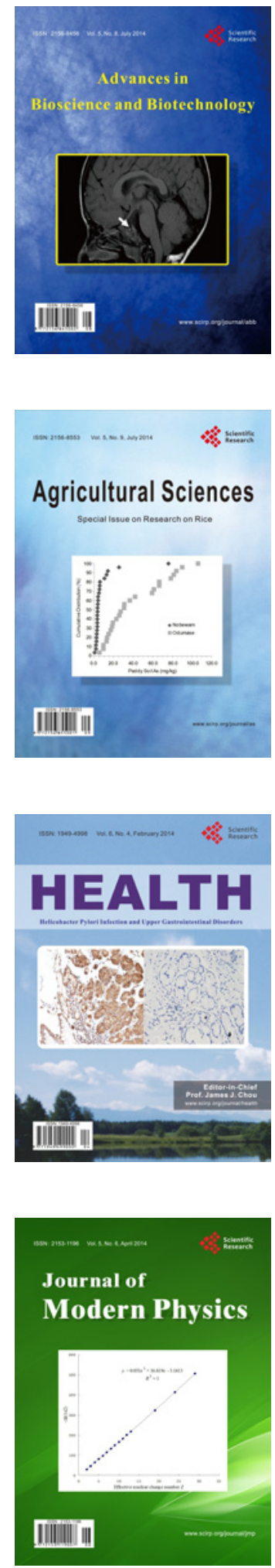
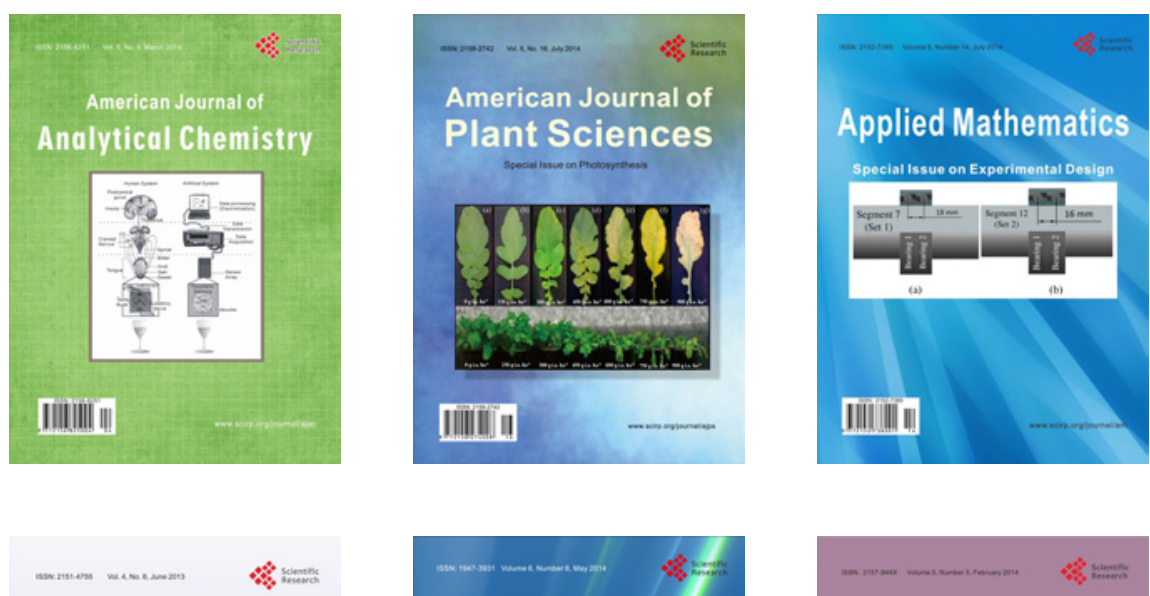

Creative Education
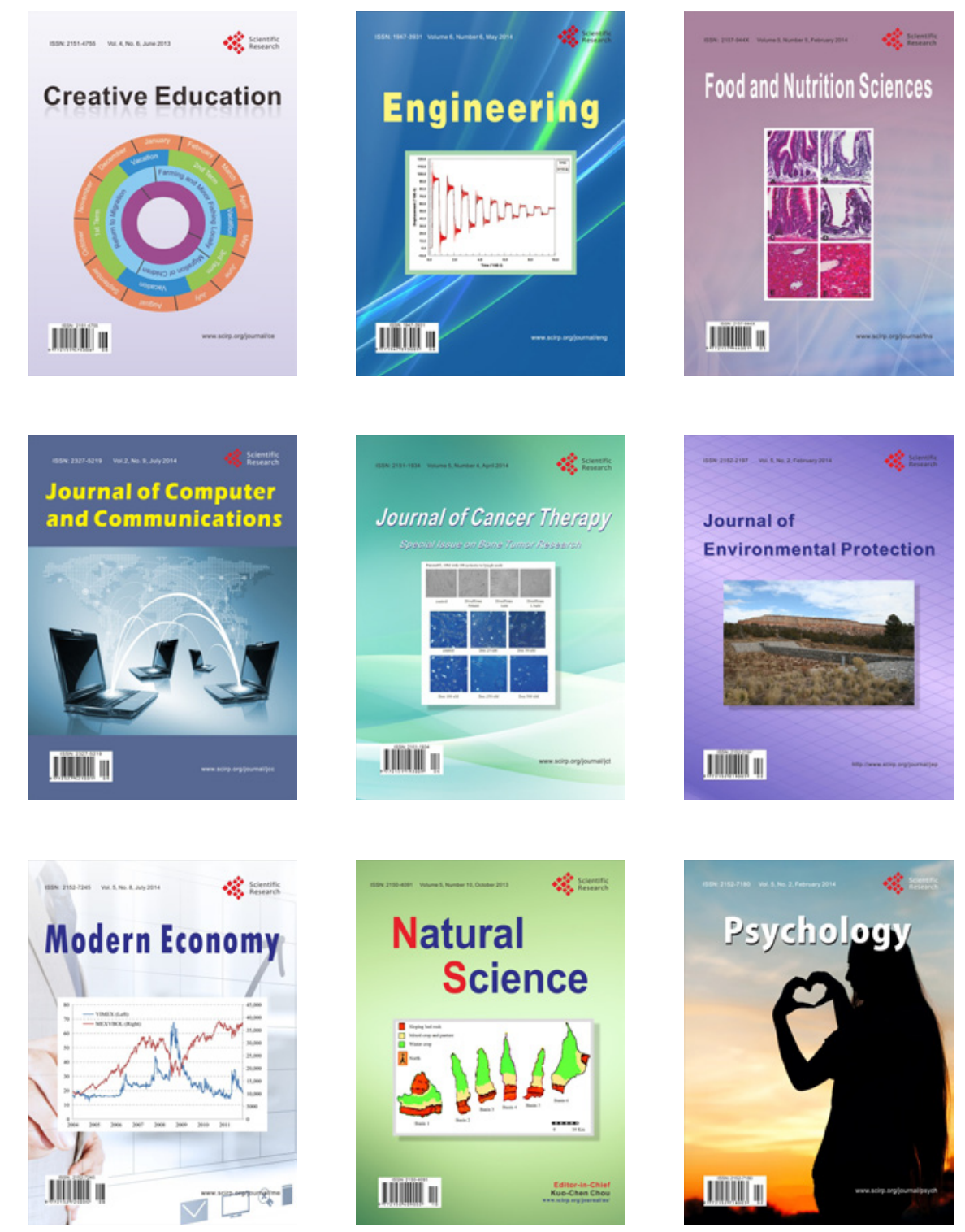